\title{
3 Research Square

\section{MEK / ERK inhibitor effectively impact generalized lymphatic anomaly (GLA) cells growth through EGFR / MEK /ERK signaling pathway}

Wa Du ( $\nabla$ wdu@bcm.edu )

Baylor College of Medicine https://orcid.org/0000-0002-1518-7301

\section{Sriram Ayyaswamy}

Baylor College of Medicine

\section{Smruti Rath}

Baylor College of Medicine

Yan Ge

Baylor College of Medicine

\section{Tommy Nguyen}

Baylor College of Medicine

Judith F Margolin

Baylor College of Medicine

\section{Debra Kearny}

Baylor College of Medicine

Thuy L Phung

Baylor College of Medicine

\section{Research}

Keywords: generalized lymphatic anomaly (GLA), primary lymphatic endothelial cell, rapamycin, dual MEK / ERK inhibitor, cell immortalization, SV40 LT

Posted Date: March 10th, 2020

DOl: https://doi.org/10.21203/rs.3.rs-16567/v1

License: (c) (i) This work is licensed under a Creative Commons Attribution 4.0 International License.

Read Full License 


\title{
MEK / ERK inhibitor effectively impact generalized lymphatic anomaly (GLA) cells growth through EGFR / MEK /ERK signaling pathway
}

\author{
Wa Du ${ }^{1 \#}$, Sriram Ayyaswamy ${ }^{1}$, Smurti Rath ${ }^{1}$, Yan Ge ${ }^{1}$, Tommy Nguyen ${ }^{1}$, Judith F. Margolin ${ }^{2}$, \\ Debra Kearny ${ }^{1}$, Thuy Phung ${ }^{1 \#}$ \\ ${ }^{1}$ Department of Pathology and Immunology, Baylor College of Medicine and Texas Children's Hospital, Houston, \\ Texas, USA; ${ }^{2}$ Department of Pediatrics, Section of Hematology-Oncology, Baylor College of Medicine and Texas \\ Children's Hospital, Houston, Texas, USA
}

\#Corresponding author

Wa Du

Department of Pathology and Immunology,

Baylor College of Medicine and Texas Children's Hospital

1102 Bates Avenue

Feigin Tower, Room 830.08

Houston, TX 77030

Phone: 832-824-0815

Email:wdu@bcm.edu

Thuy L. Phung, MD, PhD

Department of Pathology and Immunology,

Baylor College of Medicine and Texas Children's Hospital

1102 Bates Avenue

Feigin Tower, Room 830.21

Houston, TX 77030

Phone: 832-824-5202

Fax: 832-825-1165

Email: tphung@bcm.edu 


\begin{abstract}
Background

Generalized lymphatic anomaly (GLA) is characterized by diffuse or multicentric proliferation of dilated lymphatic vessels resembling common lymphatic malformation. Studies on GLA are frequently hampered by a lack of appropriate models to test the effects of potential treatments or decipher the mechanism of pathology. Moreover, diverse phenotypes observed with GLA require a large number of samples to be analyzed to obtain statistically informative results. Due to the very limited experimental material, most of the research is restricted to single case report.
\end{abstract}

\title{
Methods
}

We first time used two-step endothelial cell isolation technique (step 1: single cells were first sorted with $\alpha$ human CD31 magnetic beads; step 2: collected CD31 ${ }^{\text {Pos }}$ cells from step1 were sorted with $\alpha$-human PDPN magnetic beads) to generate two GLA-LEC cell lines, and purified normal-LEC from normal liver tissue in the same case. To characterize the aberrant phenotype of generalized lymphatic anomaly lymphatic endothelial cells (GLA-LEC\#1, and GLA-LEC\#2). We investigated GLA-LECs growth curve, cell cycle, apoptosis, and sprouting angiogenesis in vitro. Matrigel plug assay was applied in immunodeficient mice to monitor the GLA-LECs formed vasculature in vivo. Rapamycin and dual MEK / ERK inhibitor were tested to investigate the efficacy on inhibiting GLA-LEC proliferation and downstream signaling pathway.

\section{Results}

We have successfully purified GLA-LECs from GLA tissues with $>99 \%$ purity. These cells also expressed the lymphatic markers lymphatic vessel endothelial hyaluronan receptor (LYVE-1) and podoplanin (PDPN). GLALECs showed significantly higher proliferation rate compared to normal-LECs in both cases. Cell cycle analysis of cell distribution suggested that compared with normal-LECs, GLA-LECs showed increased proportion of cells in S phase and less G0/G1 phase. When GLA-LECs and normal-LECs apoptosis induced by serum deprivation, more Annexin V positive population of endothelial cells were observed in normal-LECs but not GLA-LECs. Hyperactivated epidermal growth-factor receptor (EGFR) signaling was observed in both cases of GLA-LECs, endogenously highly expression of EGF receptor and EGF induced phosphorylation of EGFR (phosphor Y1068) were found in both GLA cell lines. GLA-LECs are sensitive to both rapamycin and MEK / ERK dual inhibitor treatment. In vivo, by using Matrigel plug assay, we found both GLA-LECs and immortalized GLA-LEC (SV40) grew robust vessel-like structure.

\section{Conclusions}

In vitro, both GLA-LECs cell lines are highly proliferative as compared with normal-LECs. Rapamycin and dual MEK / ERK inhibitor dose-dependently inhibited GLA-LECs proliferation. In vivo, GLA-LECs showed angiogenic phenotype, and grew robust vessel-like structure in immunodeficient mice.

\section{Keywords}

generalized lymphatic anomaly (GLA), primary lymphatic endothelial cell, rapamycin, dual MEK / ERK inhibitor, cell immortalization, SV40 LT 


\section{Background}

Generalized lymphatic anomaly (GLA), also known as lymphangiomatosis, is a rare congenital lymphatic malformation originating from persistence of dilated lymphatics at $14-20^{\text {th }}$ week of life, and around $65 \%$ of affected patients are infants and young children [1]. GLA is characterized by diffuse or multicentric proliferation of dilated lymphatic vessels resembling common lymphatic malformation [2]. The process of lymphatic endothelial cell hyperproliferation (an abnormally high rate of cell division) results in the increase in both the size and number of lymphatic vessels observed in GLA, and usually involves multiple organs, including lungs, mediastinum, soft tissues, as well as bones and visceral organs such as spleen [3]. To date, surgical intervention such as excision, sclerotherapy, and radiation therapy are generally reserved for local control and symptom relief for GLA treatment [4-6]. Medical treatments are limited due to little knowledge we have about the aetiology of GLA. Genetic analysis revealed that phosphoinositide 3-kinase (PI3K) and NRAS somatic mutation is highly associated with this lymphatic malformation $[7,8]$. There is growing body of evidences suggested that the treatment with interferon- $\alpha-2 b$, propranolol, low-molecular weight heparin mTOR inhibitor sirolimus (rapamycin) proved to be very effective in GLA [9-14].

A rare disease is defined as affecting less than 200,000 people in the United States according to the National Institutes of Health. There are more than 7,000 rare diseases have been identified, totaling approximately 30 million patients. Studies on GLA are frequently hampered by a lack of appropriate models to test the effects of potential treatments or decipher the mechanism of pathology. Moreover, diverse phenotypes observed with GLA require a large number of samples to be analyzed to obtain statistically informative results. In the current study, we characterized the aberrant phenotype of generalized lymphatic anomaly lymphatic endothelial cells (GLA-LEC\#1, and GLA-LEC\#2) from two patients who diagnosed with GLA. We investigated GLA-LECs growth curve, cell cycle, apoptosis, and sprouting angiogenesis in vitro. Matrigel plug assay was applied in immunodeficient mice to monitor the GLA-LECs formed vasculature in vivo. mTOR inhibitor, rapamycin and dual MEK / ERK inhibitor were tested to investigate the efficacy on inhibiting GLA-LEC proliferation and downstream signaling pathway. We also immortalized one of the GLA-LEC by using SV40 LT antigen for future potential amendable drug screening and in vitro correction potential genetic mutation.

\section{Materials and Methods}

\section{Establishment of GLA lymphatic endothelial cell (GLA-LEC), normal liver lymphatic endothelial cell (Normal-LEC)}

This study was approved by the Institutional Review Boards (Baylor College of Medicine, Texas Children's Hospital) and Institutional Animal Care and Use Committee (Baylor College of Medicine). Patient characteristics are listed in Supplementary Table S1. Primary generalized lymphatic anomaly cells and normal liver lymphatic endothelial cells were purified based on previously described endothelial cell isolation method with certain modification [15]. Briefly, normal liver lymphatic endothelial cells and generalized lymphatic endothelial cells were derived from two female patients (normal-LEC\#1, GLA-LEC\#1, normal-LEC\#2, GLA-LEC\#2). Transfer to 100 
$\mathrm{mm}$ petri dish, and using sterile scissor to mince the tissue into $1 \mathrm{~mm}$ pieces. Then transfer minced tissue to $15 \mathrm{ml}$ tube, and digest the tissue with collagenase I ( $2 \mathrm{mg} / \mathrm{ml}$, LS004196, Worthington Biochemical Product) at $37^{\circ} \mathrm{C}$ with constant agitation ( $10 \mathrm{ml} / \mathrm{g}$ tissue). Following the incubation, pass the cells through $100 \mu \mathrm{m}$ and $70 \mu \mathrm{m}$ cell strainer sequentially. Spin the cells at $350 \times \mathrm{g}$ for 5 minutes, remove the supernatant and cell were first sorted by Dynabeads ${ }^{\mathrm{TM}} \mathrm{CD} 31$ Endothelial Cells (11155D, Invitrogen, $20 \mu \mathrm{l}$ beads $/ 10^{7}$ cells). Plate the CD31 ${ }^{\text {Pos }}$ cells in flasks and incubate at $37^{\circ} \mathrm{C}, 5 \% \mathrm{CO}_{2}$ in a humidified incubator. When the $\mathrm{CD} 31^{\mathrm{Pos}}$ cells reached $80-90 \%$ confluence, they were detached by $0.25 \%$ Trypsin / EDTA, washed and resuspended in $100 \mu \mathrm{PBS}$ and incubated, for 20 minutes at room temperature, with lug mouse anti-human Podoplanin (101-M40, clone 18H5, Reliatech GmbH). After incubation, cells were washed, centrifuged, resuspended in $30 \mu \mathrm{l}$ PBS $0.5 \%$ FBS, and incubated with 8ul goat-antimouse antibodies-coated magnetic beads. Then we seeded the selected CD $31^{\text {Pos Podoplanin }}{ }^{\text {Pos }}$ cells on Type I collagen (Advanced BioMatrix)-coated tissue culture dishes in Endothelial Cell Growth Medium MV2 kit (C-22121, PromoCell). The EGM-MV2 Kit contains fetal calf serum $(0.05 \mathrm{ml} / \mathrm{ml})$, human epidermal growth factor $(5 \mathrm{ng} /$ $\mathrm{ml})$, human basic fibroblast growth factor $(10 \mathrm{ng} / \mathrm{ml})$, hydrocortisone $(0.2 \mu \mathrm{g} / \mathrm{ml})$, human vascular endothelial growth factor $165(0.5 \mathrm{ng} / \mathrm{ml})$, insulin-like growth factor $(20 \mathrm{ng} / \mathrm{ml})$, and ascorbic acid $(1 \mu \mathrm{g} / \mathrm{ml})$.

\section{Cellular proliferation assay}

GLA-LEC and Normal-LEC proliferation was assessed after seeding the cells at 1,000 / well on 96-well plates in triplicates. Cells were incubated in 0.5\% FCS in EGM-MV2 basal medium for 16 hours, and then cultured in EGM-MV2 complete medium. Fluorescence ( $480 \mathrm{~nm}$ excitation and $535 \mathrm{~nm}$ emission) was measured in a microplate reader FLUOSTAR Omega (BMG LABTECH) after 2, 4, 6, 8, and 10 days, using CyQUANT Cell Proliferation Assay Kit according to manual (Invitrogen).

\section{Cell cycle and apoptosis analysis}

GLA-LECs and normal-LECs were incubated with $10 \mu \mathrm{M}$ BrdU (Sigma, B5002) for $6 \mathrm{~h}$ prior to harvesting with $0.25 \%$ trypsin / EDTA and fixing in $70 \%$ ethanol for $24 \mathrm{~h}$. Once fixed, cell pellets were acid denatured in $\mathrm{HCl}$ / Triton X-100 for 30 minutes at room temperature and neutralized with sodium tetraborate. Pellet cells were incubated with anti-BrdU antibody (MoBU-1, B35130, Thermo Fisher Scientific) conjugated to Dylight 488 for 30 minutes. Then, the cells were pelleted and incubated on ice for $1 \mathrm{~h}$ with $5 \mathrm{ng} / \mathrm{ml}$ propidium iodide and $0.1 \mu \mathrm{g} / \mathrm{ml}$ RNase A in PBS. 10,000 single cell events were analyzed by flow cytometry and gated based on forward scatter and side scatter populations, and propidium iodide staining to determine the number of cells in G1, S and G2/M phases. Cell apoptosis was evaluated using PE-Annexin V Apoptosis Detection kit I (559763, BD Biosciences) according to the manufacturer's instructions. Briefly, cells were serum-deprived for 48 hours followed by incubation in Annexin V-binding buffer containing PE-conjugated Annexin V and 7-amino-actinomycin D (7-AAD) at room temperature for 15 minutes in the dark. Labeled cells were subsequently analyzed by flow cytometry (BD Biosciences FACSCantol II).

\section{Westernblot and immunohistochemistry}

Cells were lysed with RIPA buffer (BP-115DG, Boston Bioproducts), containing a phosphatase inhibitor cocktail (Sigma). For Western blotting, $20 \mu \mathrm{g}$ protein lysates were subject to 4-15\% SDS-PAGE gels and transferred to PVDF membrane. Membranes were then blocked in 5\% nonfat dry milk / 0.1\% Tween-20 in TBS, and blotted 
with relevant antibodies against human p70 S6 kinase (CS\#9234), pS6 (CS\#4858), pMEK (CS\#9154), pERK (CS\#4370), total MEK (CS\#8727), total ERK (CS\#9102), pEGFR (Y1068, CS\#2234), total EGFR (CS\#4267), total p70 S6 kinase (CS\#2708), total S6 (\#2217), and $\beta$-Actin (A2228, sigma). Membranes were incubated with peroxidase-conjugated secondary antibodies (1:5000, Cell Signaling). Antigen-antibody complexes were visualized using ECL and chemiluminescent sensitive film (GeneMate). For immunocytochemistry and immunohistochemistry, fixed cells and cryosections were incubated with primary antibodies: mouse anti-human CD31 (14-0317-82, eBioscience); rat anti-mouse CD31 (550274, BD Pharmingen), Prox1 (NBP1-18605, Novus), LYVE-1(AB2988, Millipore), mouse anti-human podoplanin (101-M40, clone 18H5, Reliatech $\mathrm{GmbH})$, mouse anti SV40 Large T antigen (554149, BD Pharmingen). Cell nuclei were counterstained with Hoechst 33342. Fluorescent images were digitally captured with Nikon Eclipse 90i fluorescence microscope with NIS-Elements software version 4. Recombinant human epithelial grow factor (rhEGF) were purchased from R\&D (236-EG). Rapamycin (LC laboratories) and RO5126766 (Selleckchem) were dissolved in dimethyl sulfoxide (DMSO) as stock and stored at $80^{\circ} \mathrm{C}$.

\section{Fibrin Gel angiogenesis assay}

Fibrin gel angiogenesis were performed as literature described [16]. Early passage GLA-LEC\#1 and \#2 cells were coated onto Cytodex 3 microcarrier beads (Sigma) at a concentration of 400 cells / bead for 4 hours and allowed to adhere overnight. GLA-LEC coated beads were then resuspended in a $2.5 \mathrm{mg} / \mathrm{ml}$ fibrinogen solution (MP Biomedicals) at a concentration of 250 beads / $\mathrm{ml}$. Gels were formed by adding $500 \mu \mathrm{l}$ of the fibrinogen/bead suspension to each well of a 24-well plate containing $0.5 \mathrm{U}$ of thrombin (Sigma-Aldrich). Once gel clotted, $1 \mathrm{ml}$ of EGM-MV2 medium containing VEGF-C was added to each well. Assays were quantified between day 6 and 7 by live culture imaging using bright-field microscopy. At least thirty beads per condition were quantified per experiment. Numbers of branches and total length of branches were analyzed by Image J (Plugin Skeleton).

\section{In vivo Matrigel plug assay}

A total of $5 \times 10^{5}$ and / or $2 \times 10^{6}$ GLA-LECs were suspended in $200 \mu$ of ice-cold Phenol Red-free Matrigel (Corning). Implants of Matrigel alone served as controls. The mixture was transplanted subcutaneously in to the

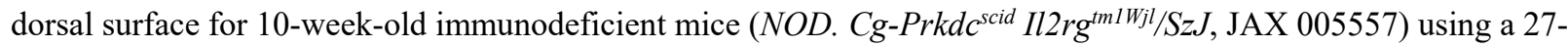
gauge needle. Two implants were injected per mouse. Mice were sacrificed on day 5 or days 10 day after injection and Matrigel plugs were removed for tissue histology and immunofluorescence staining processing.

\section{GLA-LEC immortalization}

293FT retroviral packaging cells cultured in DMEM medium with $10 \%$ fetal calf serum when transfected with pBABE-SV40 LT (Addgene \#13970) by standardized lipofectamine method [17]. Forty-eight hours after transfection, the supernatant was harvested. GLA-LEC\#1 cells cultured in EGM-MV2 were transduced with the recombinant retrovirus supernatant plug $8 \mu \mathrm{g} / \mathrm{ml}$ hexadimethrine bromide (Sigma) for 48 hours, followed by selection with $2 \mu \mathrm{g} / \mathrm{ml}$ puromycin for 14 days. Drug resistant cells were selected and expanded for further studies.

\section{Cumulative population doubling determination}

Upon subculturing, GLA-LEC\#1 and GLA-LEC\#1 (SV40) cells were plated at in initial density of $2 \times 10^{5}$ in a T25 flasks and allowed to grow. When cells reached $80 \%$ confluency, cell number was determined. This process 
was repeated up to 55 days until the cells reached 22 passages. In each passage, the passage number and date of subculturing were recorded. Population doubling time was calculated according to the equation: $3.32 \times[\log$ (the number of cells harvested)-log (the initial no of cell plated)] + S, where $S$ is the initial population doubling. Cumulative population doubling was then plotted against days in culture to study GLA-LEC\#1 and GLA-LEC\#1 (SV40) growth rate.

\section{Senescence-associated $\beta$-gal activity assay}

Cells were examined for senescence associated $\beta$-galactosidase activity [18]. GLA-LEC\#1 and GLA-LEC (SV40 LT) were continuously cultured and passaged for 15 times until reach to late passage (passage 22), then washed with phosphate buffered saline (PBS) and fixed with $2 \%$ formaldehyde, $0.2 \%$ glutaraldehyde solution in PBS at room temperature. The fixed cells were washed before addition of $1 \mathrm{mg} / \mathrm{ml} \mathrm{X}$-gal (5-bromo-4-chloro-3indolyl $\beta$-D-galactoside) in $\mathrm{N}$, N-dimethylformamide, $2 \mathrm{mM} \mathrm{MgCl}_{2}, 150 \mathrm{mM} \mathrm{NaCl}, 5 \mathrm{mM}$ potassium ferricyanide, 5 $\mathrm{mM}$ potassium ferrocyanide, $40 \mathrm{mM}$ citric acid/Na phosphate buffer, $\mathrm{pH}$ 6.0. Cells were incubated with staining solution overnight at $37^{\circ} \mathrm{C}$, nuclei stain with Hoechst 33342 for cell count and washed twice with $\mathrm{H}_{2} \mathrm{O}$ and examined under microscope. Senescent cells (stained blue) were then counted with the use of a phase-contrast microscope and their percentage determined.

\section{Statistical analysis}

Statistical analyses were performed using GraphPad Prism v5.0. Data is expressed as mean \pm SD. Unpaired two-tailed t-test were used to compared results from GLA-LEC and normal LEC group. Two-way ANOVA, followed by Bonferroni post-tests was used to compare results from GLA-LEC and normal-LEC proliferation assay. When $P$ value $<0.05$, is considered statistically significant. ${ }^{*} p<0.05,{ }^{* *} p<0.01, * * * p<0.001,{ }^{* * * *} p<0.0001$.

\section{Results}

\section{Establishment primary generalized lymphatic anomaly lymphatic endothelial cell lines (GLA-LEC)}

Lymphatic endothelial cells (LECs) from GLA lesion tissue (lung, mediastinum, stomach curvature, and pleural cavity) were successfully purified. As shown as Figure 1A, the morphology of human GLA-LEC with cobblestone-like morphology under phase-contrast microscopy. This indicated that they showed an endothelial phenotype. To ensure the purity of cells recovered by double immunomagnetic sorting, immunocytochemistry was performed on LECs cultured on glass coverslip. Here, we used three different lymphatic endothelial cell markers to verify GLA-LECs. The pan-endothelial marker CD31 (PECAM-1) is an integral membrane glycoprotein [19]. By immunoflurescence staining with CD31, we found CD31 highly enriched at both GLA-LECs cell-cell junction (Figure 1B). The transcription factor Prox1 (Prospero homeobox 1) is a marker for lymphatic endothelial cells in normal and diseased human tissue [20]. Figure 1C showed that Prox 1 was consistently found in nuclei in both cases of GLA lesion cells. LYVE-1, the lymphatic receptor for the extracellular matrix mucopolysaccharide hyaluronan $[21,22]$, was widely expressed in our GLA-LECs showing a characteristic pattern on cell membrane surface (Figure 1D). Thus, by immunocytochemistry, our population of GLA-LEC\#1 and GLA-LEC\#2 possesses the distinct phenotypic characteristics of the lymphatic endothelium. 


\section{GLA-LECs showed highly proliferative with less apoptotic phenotype in vitro}

In order to study the biological characteristics of GLA-LECs, we also purified normal liver LECs (derived from the same patient, named as normal-LEC\#1, normal-LEC\#2). Normal-LEC and GLA-LEC were seeded and let to adhere and proliferate in the presence of EGM-2MV medium (complete medium). We plate 1,000 cells / well, keep monitoring cell growth for 6 to 10 days, and every other day we added CyQUANT dye (fluorescent dye binding to cellular DNA content). We observed that at the time points examined, both GLA-LEC\#1 and GLA-LEC\#2 cells showed significantly higher proliferation (Figure $2 \mathrm{~A}, p<0.0001$; $2 \mathrm{E}, p<0.0001$ ) compared to normal-LECs in both cases. In Figure 2B, we showed analysis of cell distribution of GLA-LEC\#1 as compared with normal-LEC\#1. GLA-LEC\#1 showed less proportion of cells in G0/G1 phase ( $74.4 \%$ vs $80.6 \%, p<0.0001)$, but increased proportion of cells in $\mathrm{S}$ phase $(20.9 \%$ vs $13.8 \%, p<0.0001)$. Similarly, $\mathrm{G} 0 / \mathrm{G} 1$ phase cell proportion significantly reduced in GLA-LEC\#2 (36.4\% vs 53.2\%, $p<0.0001)$ with increased cell proportion in $\mathrm{S}$ phase $(58.1 \%$ vs $42.8 \%, p$ $<0.0001$ ) as compared to normal-LEC\#2 (Figure 2F). To investigate the cell apoptosis, GLA-LECs and normalLECs apoptosis induced by serum deprivation ( $0.5 \%$ FCS, for 48 hours) were analyzed by 7-AAD / Annexin V flow cytometry. Annexin V is a cell membrane marker for early stage apoptosis; 7-AAD stains the nucleus of apoptotic cells. Decreased Annexin V positive populations of endothelial cells were observed in GLA-LECs. Both GLALEC\#1 and GLA-LEC\#2 exhibited less apoptotic population compared with normal-LECs $(4.7 \%$ vs $8.2 \%, p<$ $0.0001 ; 14.5 \%$ vs $19.7 \%, p<0.05$, Figure $2 \mathrm{C}, 2 \mathrm{G}$ ). To explore the potential signaling pathway of GLA in both cell lines, we noticed that the endothelial cells lining the vascular channels in GLA display increased expression of epidermal growth-factor receptor (EGFR) [23]. EGFR expression and activation was examined in both GLA-LECs by western blotting. Normal-LECs showed low expression of EGFR endogenously, in contrast, both GLA-LECs highly expressed EGFR (Figure 2D, 2H). We stimulated both GLA-LEC\#1 and GLA-LEC\#2 with $100 \mathrm{ng} / \mathrm{ml}$ EGF for 10 minutes to investigate the phosphorylation of EGFR and ERK. Expectedly, both cell lines showed increased EGFR and ERK phosphorylation under EGF stimulation (Figure 2D, 2H). Taken together, both cases of GLA-LECs showed an increased cell proportion in S phase, which indicated that GLA-LECs are actively proliferating lymphatic endothelial cells. When apoptosis was induced by serum deprivation, GLA-LECs were more resistant to apoptosis and showed better survival rate. Both GLA-LEC cell lines are highly responsive to EGF stimulation, consequently EGF-induced MAPK signaling was highly activated.

\section{GLA-LEC showed increased sprouting angiogenesis in vitro}

To study GLA-LEC sprouting and branching angiogenesis, we used an in vitro angiogenesis model fibrin gel bead assay to mimic key early stages of angiogenesis (degradation of the basement membrane, sprouting of LECs into the extracellular matrix, alignment of LECs into cords, branching et al.) [16]. The same number of normal-LEC and GLA-LEC are coated onto Cytodex microcarriers and embedded into a fibrin gel. After 5 days, numerous vessels are present that can be observed under phase-contrast microscopy. As shown in Figure 3A, normal-LEC\#1 only with fewer new sprouts emerging, while GLA-LEC\#1 showed increased number of sprouts ( $7.83 \pm 2.3$ vs 16.1 $\pm 3.4, p<0.0001)$. Total length of sprout was also calculated in GLA-LEC\#1 and normal-LEC\#1 (350.4 \pm 157.2 vs $217.1 \pm 113.9, p<0.0001$ ) (Figure3B). In another GLA cell line, GLA-LEC\#2, we also found the similar results by 
using this three-dimensional fibrin gel bead assay. Quantification of vessel sprouts showed that GLA-LEC\#2 had stronger capacity in tube formation as compared to normal-LEC\#2 (73.8 \pm 41.1 vs $29.6 \pm 22.0, p<0.0001$, Figure $3 \mathrm{C}$ and 3D) with increased total length of sprouts $(476.9 \pm 230.0$ vs $184.6 \pm 124.5, p<0.0001)$.

\section{In vivo angiogenesis of GLA-LEC}

The in vivo angiogenesis potential of GLA-LEC\#1 was determined by Matrigel plug assay. GLA-LEC\#1 cells

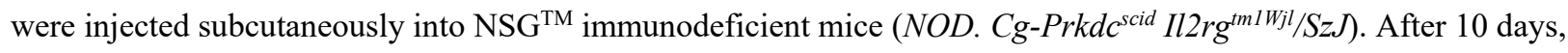
Matrigel plugs were removed from both control (Matrigel alone) and GLA-LEC\#1 group (Figure 4A). Hematoxylin and Eosin staining revealed that grafts with GLA-LEC\#1 contain robust vessel-like structure (Figure 4B), but not in Matrigel alone. The presence of luminal murine erythrocytes suggested that the newly formed vessel-like structures were perfused within 10 days. In Figure 4C, to identify the GLA-LEC\#1, immunoflurescence staining by antihuman CD31 (in green) and anti-mouse CD31 (in red) antibodies were conducted. In most of the vessel-like structure, endothelial cells are patient derived GLA-LEC\#1, but not host derived mouse endothelial cells. This data indicated that GLA-LEC showed higher viability and angiogenic phenotype in vivo.

\section{GLA-LEC is sensitive to Rapamycin and MEK / ERK dual inhibitor treatment}

Next, we examined whether rapamycin, which is broadly used in GLA treatment, may affect GLA-LEC viability. We gave rapamycin at different doses $(10 \mathrm{ng} / \mathrm{ml}, 25 \mathrm{ng} / \mathrm{ml}, 50 \mathrm{ng} / \mathrm{ml}, 100 \mathrm{ng} / \mathrm{ml})$ to our generated two GLA-LEC cell lines, GLA-LEC\#1 and GLA-LEC\#2. We found that rapamycin dose-dependently inhibited GLALEC\#1 proliferation in CyQUANT assay (Figure 5A). GLA-LEC\#1 proliferation was completely inhibited at 100 $\mathrm{ng} / \mathrm{ml}$ of rapamycin treatment. In GLA-LEC\#2, minimum dose of rapamycin can strongly inhibit the cell growth (Figure 5E), and cell growth is completely inhibited by $25 \mathrm{ng} / \mathrm{ml}$ rapamycin treatment. It also suggested that GLALECs showed sensitivity diversity on rapamycin treatment. We also studied downstream signaling pathway when GLA-LECs were treated by rapamycin. p70 ribosomal S6 kinase (S6K1) is a major substrate of the mammalian target of rapamycin (mTOR) kinase. In both GLA-LECs, rapamycin $(25 \mathrm{ng} / \mathrm{ml})$ significantly decreased S6K1 and S6 phosphorylation detected by western blotting (Figure 5C and 5G). This data indicated that besides of p70 S6 kinase signaling pathway, there could be other signaling pathway is also involved in GLA-LECs proliferation. Then, we investigated the effect of MEK / ERK dual inhibitor RO5126766 (or CH5126766) on cell growth inhibition in both GLA-LEC\#1 and GLA-LEC\#2. In both cell lines, with addition of RO5126766 (10nM, 50nM, 250nM, 1000nM) dose dependently inhibited both GLA-LEC\#1 and GLA-LEC\#2 growth curve (Figure 5B and 5F). Western blotting data suggested that both phosphorylation of MEK and ERK in GLA-LECs were dose-dependently inhibited by RO5126766 treatment (Figure 5D and 5H). The inhibition of cell growth induced by MEK / ERK inhibitor is consistent with inhibition of phosphorylation MEK and ERK in both patient derived GLA-LEC cell lines. Taken together, mTOR and MEK / ERK pathway inhibitor showed high efficiency on GLA-LECs proliferation inhibition.

\section{GLA-LEC is immortalized for in vitro and in vivo study}

It is important to establish a good and reproducible model that has the potential for multiple application, in particular a model of a rare disease such as generalized lymphatic anomaly. The commonly used vector for immortalization is SV40 LT, which exhibits immortalization properties without fully transforming the cells. In the 
current study, we used a retrovirus to overexpress SV40 T antigen in GLA-LEC\#1. Positive single cell clone after puromycin selection is subcultured and expanded. Both immunocytochemistry and western blotting were used to determine SV40 large T expression in the generated cell line (Figure 6A and 6B). SV40 large T antigen specifically expressed in cell nuclei. Figure 6C indicated the cumulated population doubling levels of the parental GLA-LEC\#1 compared with GLA-LEC\#1 (SV40). GLA-LEC\#1 (SV40) grew much faster than parental cells. Later passage, SV40 LT antigen is still can be detected and stably expressed in the immortalized GLA-LEC (SV40) cell line as shown in Figure 6D. We next investigated the cellular senescence in GLA-LEC\#1. In later passage (passage 22), GLA-LEC\#1 showed increased number of SA- $\beta$-gal-stained cells, whereas the GLA-LEC (SV 40) did not show any positive staining (Figure 6E). This data indicated that there are large amount primary GLA-LEC\#1 being senescence at late passage. Senescence of GLA-LEC\#1 (SV40) is undetectable even after long time subculture or late passage. In vivo, we also tested GLA-LEC (SV40) viability by using Matrigel plug assay. With different cell density $\left(0.5 \times 10^{6}\right.$ and $\left.2 \times 10^{6}\right)$, different time point (Day5 and Day10, Figure 6F), GLA-LEC (SV40) showed robust vessellike structures detected by anti-human CD31 antibody immunoflurescence staining which is similar to parental GLA-LEC\#1(Figure 6G). We did not found transformed cell colony in this in vivo angiogenesis model.

\section{Discussions}

Generalized lymphatic anomaly is an extremely rare disease. Due to the very limited experimental material, most of the research is restricted to single case report. In this study, we first time used two-step endothelial cell isolation technique (step 1: single cells were first sorted with $\alpha$-human CD31 magnetic beads; step 2: collected CD31 $1^{\text {Pos }}$ cells from step1 were sorted with $\alpha$-human PDPN magnetic beads), and we successfully generated two GLA-LEC cell lines. Meanwhile, we also purified the normal-LEC from normal liver tissue in the same case. These paired primary cells obtained from same case benefited the researcher to have the proper control sample in GLA study.

In vitro, we found GLA-LECs showed highly proliferative phenotype as compared with normal-LECs. Similar results which demonstrated that lymphatic endothelial cells from pediatric patients with GLA showed a high proliferation rate was also reported by Mori et al [24]. We also found that both GLA-LECs showed decreased cell portion in G0/G1 phase with increased cell portion in S phase, but we did not find difference in G2/M phase. The cell cycle analysis results suggested that GLA-LECs increased S phase with less G0/G1 phase was consistently associated with GLA-LEC highly proliferative phenotype. In addition, we found GLA-LEC is more resistant to serum deprivation induced apoptosis. As we known, hyperactive mTORC1 signaling is a major contributor to growth factor deprivation-induced apoptosis resistance. The inhibition of mTORC1 by rapamycin could be the best therapeutic strategy for GLA as far as new mechanism will be elucidated.

Moreover, GLA-LECs generate capillary-like structures containing a lumen when seeded on Cytodex beads. The morphological quantification of the vascular network was achieved through a newly developed automatic method suitable for image J. The enhanced capacity of GLA-LECs in the three-dimensional sprouting angiogenesis assay affected by two main parameters of the vascular network: the number of sprouts, and the total length of the capillary network. 
Up to date, there are various somatic mutations were found in GLA. PIK3CA, NRAS, and recently ARAF recurrent mutation was identified in GLA patients [25]. But in many cases of GLA, the somatic mutation is still undetectable. Here, we first time reported that these two GLA-LEC cell lines are sensitive and dose-dependently response to rapamycin and dual MEK / ERK inhibitor antiproliferative treatment. Lymphatic endothelial cell ERK activation is responsible for the lymphatic phenotype [26], suppression of ERK activation would be the other therapeutic benefit besides mTOR inhibitor.

In the current study, we generated two primary GLA lymphatic endothelial cell lines from this rare disorder. Isolated primary parental GLA-LEC will be senescent after long term culture, with SV40 LT immortalized GLALEC (SV40) showed rapid growth and had the advantage to maintain a stable cellular phenotype. Once cell lines are immortalized, they are amenable to many downstream applications, including drug screening and in vitro correction of the genetic mutation, or in vivo study by using immunodeficient mice. The generation of patient derived specific cell lines provided an invaluable tool for translational research, especially in rare disease.

\section{Conclusions}

Taken together, we reported the first generalized lymphatic anomaly cell lines two-step cell isolation technique. GLA-LECs were characterized by series lymphatic endothelial cell marker immunofluorescent staining (CD31, LYVE-1 and Prox1). In vitro, GLA-LECs are highly proliferative and less apoptotic compared to same patient derived normal-LECs. GLA-LECs are more responsive to EGF stimulation, resulted in activation of EGFR / MEK / ERK signaling pathway. Both rapamycin and MEK / ERK dual inhibitor can inhibit GLA-LECs growth dose dependently. In vivo, by using the novel extremely immunodeficient mouse model ( $\mathrm{NSG}^{\mathrm{TM}}$ ), GLA-LECs grew rapidly in Matrigel plug assay, we can track the human derived GLA-LECs and host endothelial cell by anti-human and anti-mouse CD31, respectively. We also immortalized GLA-LECs by using Simian virus 40 large $\mathrm{T}$ antigen for future drug screening and in vitro correction of the genetic mutation research. 


\author{
Abbreviations \\ LEC: lymphatic endothelial cell \\ PDPN: podoplanin \\ LYVE-1: lymphatic vessel endothelial receptor 1 \\ CD31: cluster of differentiation 31 \\ H\&E: Hematoxylin and eosin \\ SV40 LT: Simian virus 40 large T antigen \\ mTOR: mammalian target of rapamycin \\ mTORC1: mammalian target of rapamycin complex 1 \\ ERK: extracellular signal regulated kinase \\ EGFR: epidermal growth-factor receptor
}


Figure1. Establishment primary generalized lymphatic anomaly lymphatic endothelial cell lines (GLA-LEC). (A) Confluent GLA-LEC\#1 and GLA-LEC\#2 viewed under phase-contrast microscopy exhibit the typical cobblestone morphology. (B) Fluorescence microscopy showing positive immunoreactivity for CD31, Prox-1 (C), and LYVE-1 (D). Scale bar, $100 \mu \mathrm{m}$.

Figure 2. GLA-LECs showed highly proliferative with less apoptotic phenotype in vitro (A) Cell proliferation of Normal-LEC\#1 and GLA-LEC\#1 was examined by CyQUANT. 1,000 cells (in 96-well plate) are seeded per well, every other day fluorescent dye binding to cellular DNA content was added to each well. Signal was collected as fluorescent unit. ${ }^{* *} p<0.01,{ }^{* * *} p<0.0001, \mathrm{~N}=3$. (B) Normal-LEC\#1 and GLA-LEC\#1 cell populations residing in G0/G1, S and G2/M. ****p $<0.0001, \mathrm{~N}=8$. (C) Normal-LEC\#1 and GLA-LEC\#1cells were serum-deprived for 48 hours followed by incubation in Annexin V-binding buffer containing PE-conjugated Annexin V and 7-aminoactinomycin D (7-AAD) at room temperature for 15 minutes in the dark. Labeled cells were subsequently analyzed by flow cytometry. ${ }^{* * * *} p<0.0001, \mathrm{~N}=6$. (D) Western blot for phosphorylated and total EGFR and ERK in GLALEC\#1 serum-starved overnight and exposed to $100 \mathrm{ng} / \mathrm{ml}$ EGF for 10 minutes. $\beta$-actin is used as loading control. (E) Cell proliferation of Normal-LEC\#2 and GLA-LEC\#2 was examined by CyQUANT. (F) Normal-LEC\#2 and GLA-LEC\#2 cell populations residing in G0/G1, $\mathrm{S}$ and G2/M. **** $p<0.0001, \mathrm{~N}=3$. (G) Normal-LEC\#2 and GLA-LEC\#2 cells were serum-deprived for 48 hours followed by Annexin V / 7-AAD incubation, subjected by flow cytometry. ${ }^{*} p<0.05, \mathrm{~N}=4$. (H) Western blot for phosphorylated and total ERK in GLA-LEC\#2 serum-starved overnight and exposed to $100 \mathrm{ng} / \mathrm{ml} \mathrm{EGF}$ for 10 minutes.

Figure 3. GLA-LECs showed increased sprouting angiogenesis in vitro (A) Photomicrographs of normal-LEC\#1 and GLA-LEC\#1 on Cytodex microcarriers in three-dimensional fibrin gels. (B) Number of sprouts and total length of sprouts were quantified by ImageJ. $* * * * p<0.0001, N=25$. (C) Photomicrographs of normal-LEC\#2 and GLALEC\#2 on Cytodex microcarriers in three-dimensional fibrin gels. (D) Quantitative analysis of number of sprouts and total length of sprouts. $* * * * p 0.0001, \mathrm{~N}=30$. Scale $\mathrm{bar}=150 \mu \mathrm{m}$.

Figure 4. In vivo angiogenesis assay of GLA-LEC. (A) GLA-LEC\#1 $\left(2 \times 10^{6}\right)$ was injected subcutaneously into


control (Matrigel alone) and GLA-LEC\#1 group. (B) Hematoxylin and Eosin staining revealed that grafts with GLA-LECs contain robust vessel-like structure, but not in control sample. (C) To identify the patient derived GLALEC\#1, immunoflurescence staining by anti-human CD31 (in green) and anti-mouse CD31 (in red) antibodies were conducted. In most of the vessel-like structure, endothelial cells are patient derived GLA-LEC\#1, but not host derived mouse endothelial cells.

Figure 5. GLA-LECs are sensitive to Rapamycin and MEK / ERK dual inhibitor treatment. (A, E) GLALEC\#1 and GLA-LEC\#2 were treated by different dose of rapamycin $(10 \mathrm{ng} / \mathrm{ml}, 25 \mathrm{ng} / \mathrm{ml}, 50 \mathrm{ng} / \mathrm{ml}, 100 \mathrm{ng} / \mathrm{ml})$. CyQUANT assay was used to determine the cell proliferation. ${ }^{* * *} p<0.001, \mathrm{~N}=3$. (C, G) Western blotting was 
applied to study downstream signaling pathway involved in rapamycin treatment. (B, F) GLA-LEC\#1 and \#2 were treated by different dose of dual MEK / ERK inhibitor RO5126766 (10nM, 50nM, 250nM, 1000nM). CyQUANT assay was used to determine the cell proliferation. $* * * p<0.001, \mathrm{~N}=3$. Western blotting was applied to investigate the downstream signaling pathway involved in MEK / ERK inhibitor treatment.

Figure 6. GLA-LEC\#1 immortalized with SV40 large T antigen. (A, B) Retrovirus was used to overexpress SV40 T antigen in GLA-LEC\#1. Both immunocytochemistry and western blotting were used to determine SV40 large $\mathrm{T}$ expression in the generated cell line. (C) Cumulated population doubling levels of the parental GLA-LEC\#1 compared with GLA-LEC\#1 (SV40). (D) Later passage (passage 22), SV40 LT antigen is still can be detected and stably expressed in the immortalized GLA-LEC (SV40) cell line. (E) In later passage (passage 22), GLA-LEC\#1 showed increased number of SA- $\beta$-gal-stained cells, whereas the GLA-LEC (SV 40) did not show any positive staining. (F, G) In vivo GLA-LEC (SV40) Matrigel plug assay. With different cell density $\left(0.5 \times 10^{6}\right.$ and $\left.2 \times 10^{6}\right)$, different time point (Day5 and Day10), GLA-LEC (SV40) showed robust vessel-like structures detected by antihuman CD31 antibody immunoflurescence staining. Scale bar, $100 \mu \mathrm{m}$. 


\section{References}

[1] S.W.W. F. M. Enzinger, "Tumors of lymphatic vessels", in Soft Tissue Tumors, (1994) 679-700.

[2] M. Ozeki, A. Fujino, K. Matsuoka, S. Nosaka, T. Kuroda, T. Fukao, Clinical Features and Prognosis of Generalized Lymphatic Anomaly, Kaposiform Lymphangiomatosis, and Gorham-Stout Disease, Pediatr Blood Cancer, 63 (2016) 832-838.

[3] F. Luisi, O. Torre, S. Harari, Thoracic involvement in generalised lymphatic anomaly (or lymphangiomatosis), Eur Respir Rev, 25 (2016) 170-177.

[4] E.J. Bermejo Casero, R. Mongil Poce, R. Arrabal Sanchez, A. Fernandez de Rota Avecilla, A. Benitez Domenech, J.L. Fernandez Bermudez, [Diffuse thoracic lymphangiomatosis: diagnosis and treatment], Arch Bronconeumol, 40 (2004) 599-601.

[5] A. Kandil, A.Y. Rostom, W.A. Mourad, Y. Khafaga, A.R. Gershuny, G. el-Hosseiny, Successful control of extensive thoracic lymphangiomatosis by irradiation, Clin Oncol (R Coll Radiol), 9 (1997) 407-411.

[6] T. Takemura, M. Watanabe, K. Takagi, S. Tanaka, S. Aida, Thoracoscopic resection of a solitary pulmonary lymphangioma: report of a case, Surg Today, 25 (1995) 651-653.

[7] E. Manevitz-Mendelson, G.S. Leichner, O. Barel, I. Davidi-Avrahami, L. Ziv-Strasser, E. Eyal, I. Pessach, U. Rimon, A. Barzilai, A. Hirshberg, K. Chechekes, N. Amariglio, G. Rechavi, K. Yaniv, S. Greenberger, Somatic NRAS mutation in patient with generalized lymphatic anomaly, Angiogenesis, 21 (2018) 287-298.

[8] L. Rodriguez-Laguna, N. Agra, K. Ibanez, G. Oliva-Molina, G. Gordo, N. Khurana, D. Hominick, M. Beato, I. Colmenero, G. Herranz, J.M. Torres Canizalez, R. Rodriguez Pena, E. Vallespin, R. Martin-Arenas, A. Del Pozo, C. Villaverde, A. Bustamante, C. Ayuso, P. Lapunzina, J.C. Lopez-Gutierrez, M.T. Dellinger, V. Martinez-Glez, Somatic activating mutations in PIK3CA cause generalized lymphatic anomaly, J Exp Med, 216 (2019) 407-418. [9] A. Bassi, S. Syed, Multifocal infiltrative lymphangiomatosis in a child and successful treatment with sirolimus, Mayo Clin Proc, 89 (2014) e129.

[10] N. Laforgia, F. Schettini, D. De Mattia, D. Martinelli, G. Ladisa, V. Favia, Lymphatic Malformation in Newborns as the First Sign of Diffuse Lymphangiomatosis: Successful Treatment with Sirolimus, Neonatology, 109 (2016) 52-55.

[11] Y. Luo, L. Liu, D. Rogers, W. Su, Y. Odaka, H. Zhou, W. Chen, T. Shen, J.S. Alexander, S. Huang, Rapamycin inhibits lymphatic endothelial cell tube formation by downregulating vascular endothelial growth factor receptor 3 protein expression, Neoplasia, 14 (2012) 228-237.

[12] J. Reinglas, R. Ramphal, M. Bromwich, The successful management of diffuse lymphangiomatosis using sirolimus: a case report, Laryngoscope, 121 (2011) 1851-1854.

[13] R. Venkatramani, N.S. Ma, P. Pitukcheewanont, M.H. Malogolowkin, L. Mascarenhas, Gorham's disease and diffuse lymphangiomatosis in children and adolescents, Pediatr Blood Cancer, 56 (2011) 667-670.

[14] Z. Wang, K. Li, W. Yao, K. Dong, X. Xiao, S. Zheng, Successful treatment of kaposiform lymphangiomatosis with sirolimus, Pediatr Blood Cancer, 62 (2015) 1291-1293. 
[15] W. Du, D. Gerald, C.A. Perruzzi, P. Rodriguez-Waitkus, L. Enayati, B. Krishnan, J. Edmonds, M.L. Hochman, D.C. Lev, T.L. Phung, Vascular tumors have increased p70 S6-kinase activation and are inhibited by topical rapamycin, Lab Invest, 93 (2013) 1115-1127.

[16] M.N. Nakatsu, C.C. Hughes, An optimized three-dimensional in vitro model for the analysis of angiogenesis, Methods Enzymol, 443 (2008) 65-82.

[17] J.J. Zhao, O.V. Gjoerup, R.R. Subramanian, Y. Cheng, W. Chen, T.M. Roberts, W.C. Hahn, Human mammary epithelial cell transformation through the activation of phosphatidylinositol 3-kinase, Cancer Cell, 3 (2003) 483-495. [18] G.P. Dimri, X. Lee, G. Basile, M. Acosta, G. Scott, C. Roskelley, E.E. Medrano, M. Linskens, I. Rubelj, O. Pereira-Smith, et al., A biomarker that identifies senescent human cells in culture and in aging skin in vivo, Proc Natl Acad Sci U S A, 92 (1995) 9363-9367.

[19] P. Lertkiatmongkol, D. Liao, H. Mei, Y. Hu, P.J. Newman, Endothelial functions of platelet/endothelial cell adhesion molecule-1 (CD31), Curr Opin Hematol, 23 (2016) 253-259.

[20] J. Wilting, M. Papoutsi, B. Christ, K.H. Nicolaides, C.S. von Kaisenberg, J. Borges, G.B. Stark, K. Alitalo, S.I. Tomarev, C. Niemeyer, J. Rossler, The transcription factor Prox 1 is a marker for lymphatic endothelial cells in normal and diseased human tissues, FASEB J, 16 (2002) 1271-1273.

[21] S. Podgrabinska, P. Braun, P. Velasco, B. Kloos, M.S. Pepper, M. Skobe, Molecular characterization of lymphatic endothelial cells, Proc Natl Acad Sci U S A, 99 (2002) 16069-16074.

[22] D.G. Jackson, R. Prevo, S. Clasper, S. Banerji, LYVE-1, the lymphatic system and tumor lymphangiogenesis, Trends Immunol, 22 (2001) 317-321.

[23] H. Mertsching, T. Walles, G. Bakker, I. Wildfang, P. Macchiarini, Expression of epidermal growth-factor receptor in lymphangiomatosis: a new therapeutic target?, Lancet Oncol, 5 (2004) 353.

[24] M. Mori, M. Dictor, N. Brodszki, J.C. Lopez-Gutierrez, M. Beato, J.S. Erjefalt, E.A. Eklund, Pulmonary and pleural lymphatic endothelial cells from pediatric, but not adult, patients with Gorham-Stout disease and generalized lymphatic anomaly, show a high proliferation rate, Orphanet J Rare Dis, 11 (2016) 67.

[25] D. Li, M.E. March, A. Gutierrez-Uzquiza, C. Kao, C. Seiler, E. Pinto, L.S. Matsuoka, M.R. Battig, E.J. Bhoj, T.L. Wenger, L. Tian, N. Robinson, T. Wang, Y. Liu, B.M. Weinstein, M. Swift, H.M. Jung, C.N. Kaminski, R. Chiavacci, J.A. Perkins, M.A. Levine, P.M.A. Sleiman, P.J. Hicks, J.T. Strausbaugh, J.B. Belasco, Y. Dori, H. Hakonarson, ARAF recurrent mutation causes central conducting lymphatic anomaly treatable with a MEK inhibitor, Nat Med, 25 (2019) 1116-1122.

[26] Y. Deng, D. Atri, A. Eichmann, M. Simons, Endothelial ERK signaling controls lymphatic fate specification, J Clin Invest, 123 (2013) 1202-1215. 


\section{Declarations}

\section{Echics approval and consent to participate}

Not applicable.

\section{Consent for publication}

No applicable.

\section{Availability of data and material}

Not applicable

\section{Competing interests}

All the authors declare that they do not have any competing or financial interests.

\section{Funding}

The project received no direct funding.

\section{Authors' contributions}

WD and TP contributed to the experiment design, and interpretation. WD, SA, SR, YG and TN performed the experiments. WD, SR and YG analyzed the data. WD wrote the manuscript. JM and DK performed the sample collection. All authors had final approval of the submitted and published versions.

\section{Acknowledgement}

We thank Dr. Elisa Boscolo (Cincinnati Children's Hospital) for providing Matrigel plug tissue processing protocol.

\section{Author information}

\section{Affiliations}

Department of Pathology and Immunology, Baylor College of Medicine and Texas Children's Hospital, Houston, Texas, USA

Wa Du, Sriram Ayyaswamy, Smurti Rath, Yan Ge, Tommy Nguyen, Debra Kearny, Thuy Phung,

Department of Pediatrics, Section of Hematology-Oncology, Baylor College of Medicine and Texas Children's Hospital, Houston, Texas, USA

Judith F. Margolin

\section{Corresponding author}

Correspondence to Wa Du and Thuy L. Phung. 


\section{Figures}



Figure 1

Establishment primary generalized lymphatic anomaly lymphatic endothelial cell lines (GLA-LEC). (A) Confluent GLA-LEC\#1 and GLA-LEC\#2 viewed under phase-contrast microscopy exhibit the typical cobblestone morphology. (B) Fluorescence microscopy showing positive immunoreactivity for CD31, Prox-1 (C), and LYVE-1 (D). Scale bar, 1000m. 
Figure 2

A

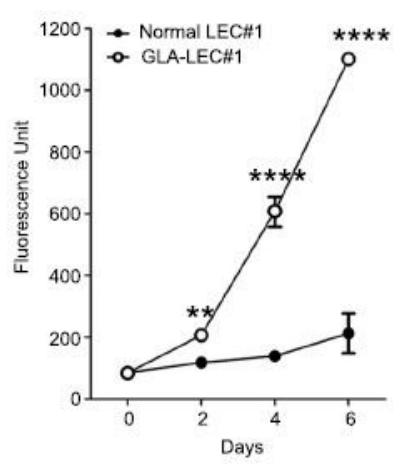

$\mathrm{E}$

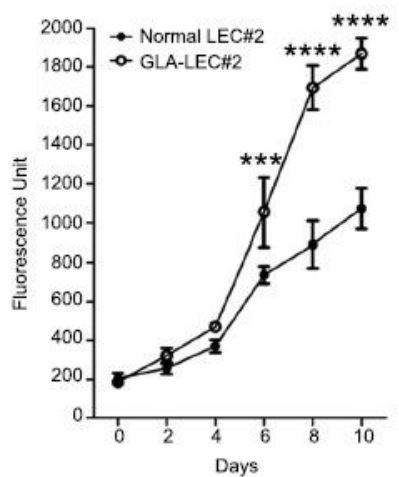

B

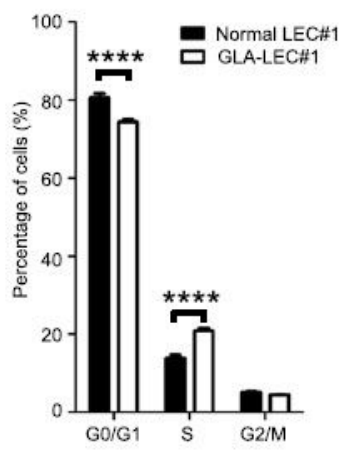

$\mathrm{F}$

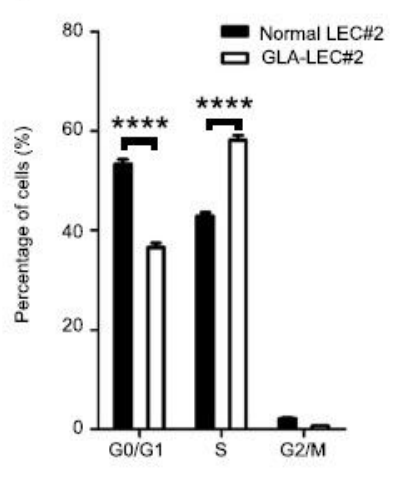

C

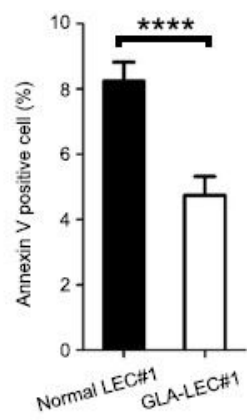

G

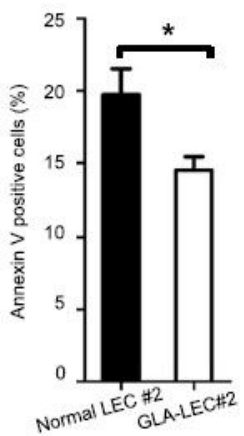

D

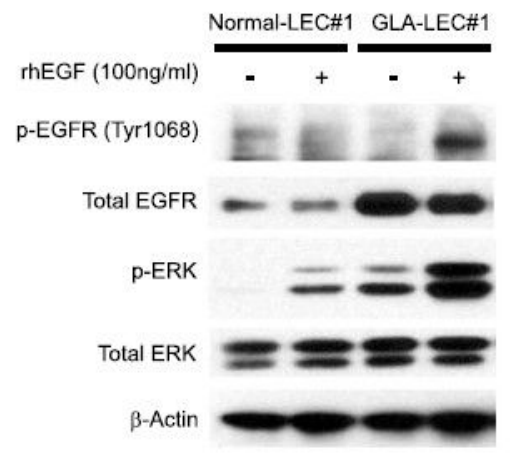

H

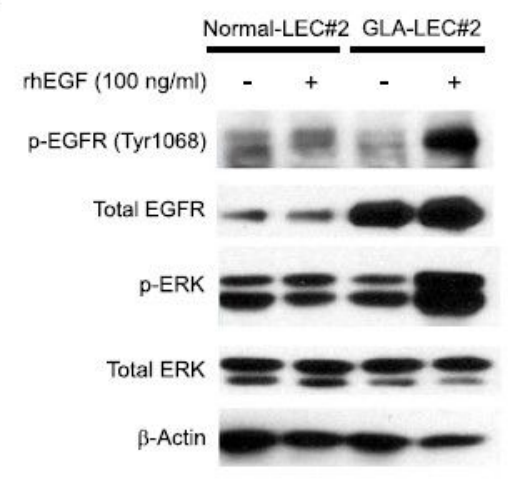

\section{Figure 2}

GLA-LECs showed highly proliferative with less apoptotic phenotype in vitro (A) Cell proliferation of Normal-LEC\#1 and GLA-LEC\#1 was examined by CyQUANT. 1,000 cells (in 96-well plate) are seeded per well, every other day fluorescent dye binding to cellular DNA content was added to each well. Signal was collected as fluorescent unit. ${ }^{*} \mathrm{p}<0.01,{ }^{\star \star \star \star} \mathrm{p}<0.0001, \mathrm{~N}=3$. (B) Normal-LEC\#1 and GLA-LEC\#1 cell populations residing in $\mathrm{G0} / \mathrm{G} 1, \mathrm{~S}$ and $\mathrm{G} 2 / \mathrm{M}$. ${ }^{\star \star * \star} \mathrm{p}<0.0001, \mathrm{~N}=8$. (C) Normal-LEC\#1 and GLA-LEC\#1cells were serum-deprived for 48 hours followed by incubation in Annexin V-binding buffer containing PEconjugated Annexin $V$ and 7-aminoactinomycin $D(7-A A D)$ at room temperature for 15 minutes in the

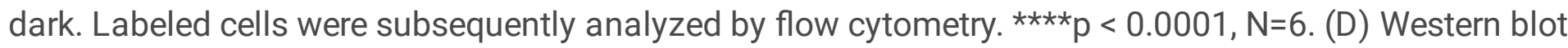
for phosphorylated and total EGFR and ERK in GLALEC\# 1 serum-starved overnight and exposed to 100 $\mathrm{ng} / \mathrm{ml}$ EGF for 10 minutes. -actin is used as loading control. (E) Cell proliferation of Normal-LEC\#2 and GLA-LEC\#2 was examined by CyQUANT. (F) Normal-LEC\#2 and GLA-LEC\#2 cell populations residing in G0/G1, S and G2/M. ${ }^{\star \star \star *} p<0.0001, N=3$. (G) Normal-LEC\#2 and GLA-LEC\#2 cells were serum-deprived for 48 hours followed by Annexin V / 7-AAD incubation, subjected by flow cytometry. ${ }^{*} p<0.05, N=4$. $(H)$ Western blot for phosphorylated and total ERK in GLA-LEC\#2 serum-starved overnight and exposed to $100 \mathrm{ng} / \mathrm{ml}$ EGF for 10 minutes. 
Figure 3

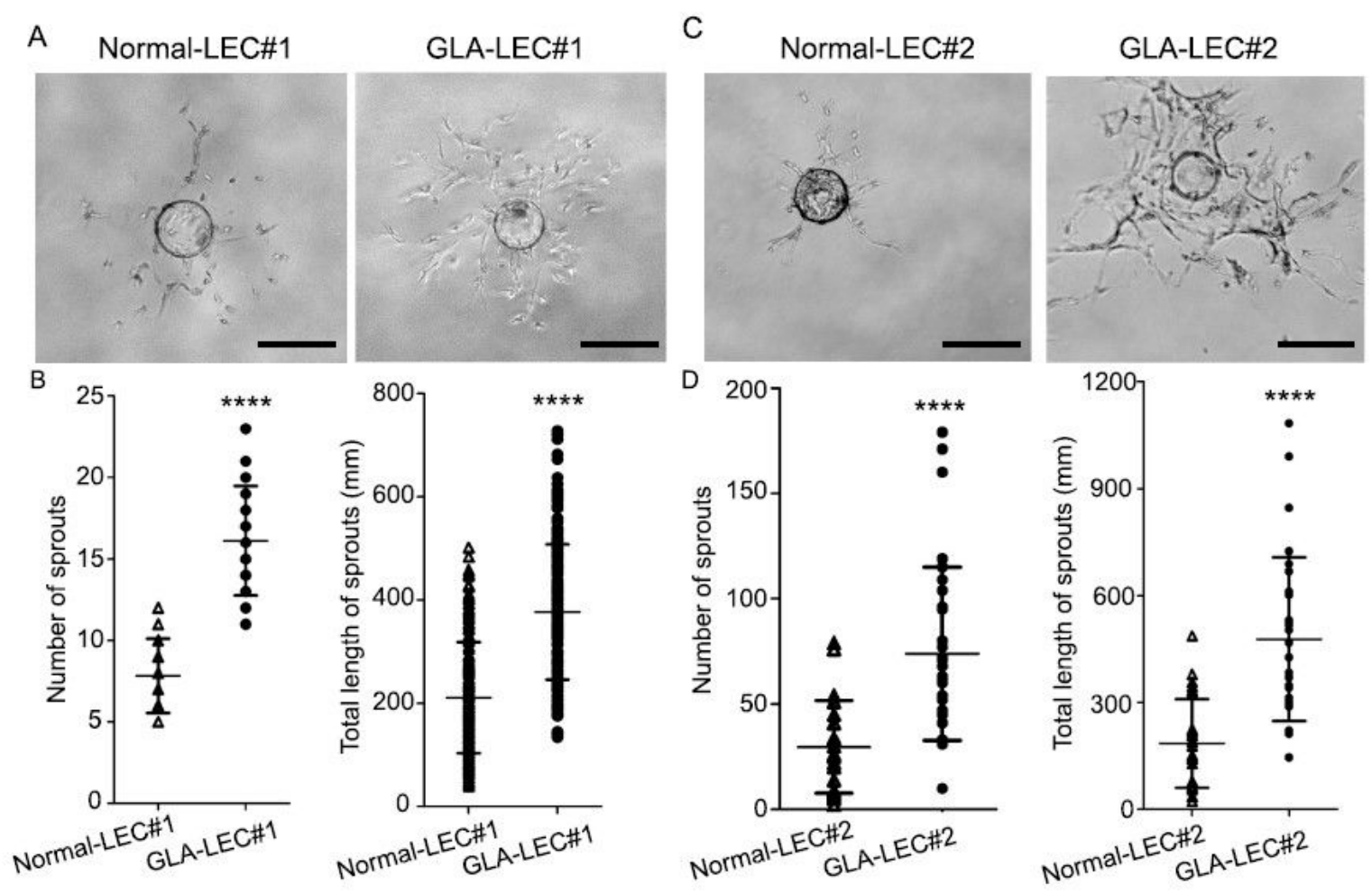

Figure 3

GLA-LECs showed increased sprouting angiogenesis in vitro (A) Photomicrographs of normal-LEC\#1 and GLA-LEC\#1 on Cytodex microcarriers in three-dimensional fibrin gels. (B) Number of sprouts and total length of sprouts were quantified by ImageJ. ${ }^{\star \star \star \star} p<0.0001, N=25$. (C) Photomicrographs of normalLEC\#2 and GLALEC\# 2 on Cytodex microcarriers in three-dimensional fibrin gels. (D) Quantitative analysis of number of sprouts and total length of sprouts. ${ }^{\star \star *} \mathrm{p}<0.0001, \mathrm{~N}=30$. Scale bar $=150 \mathrm{Dm}$. 
Figure 4

A

\section{Control GLA-LEC\#1}

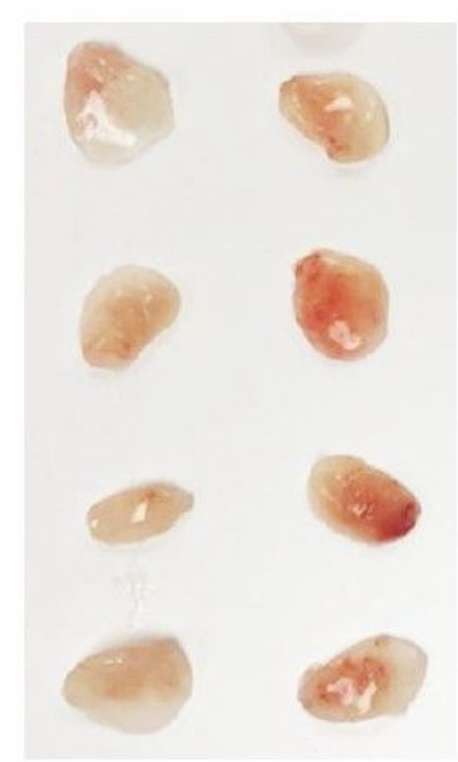

B

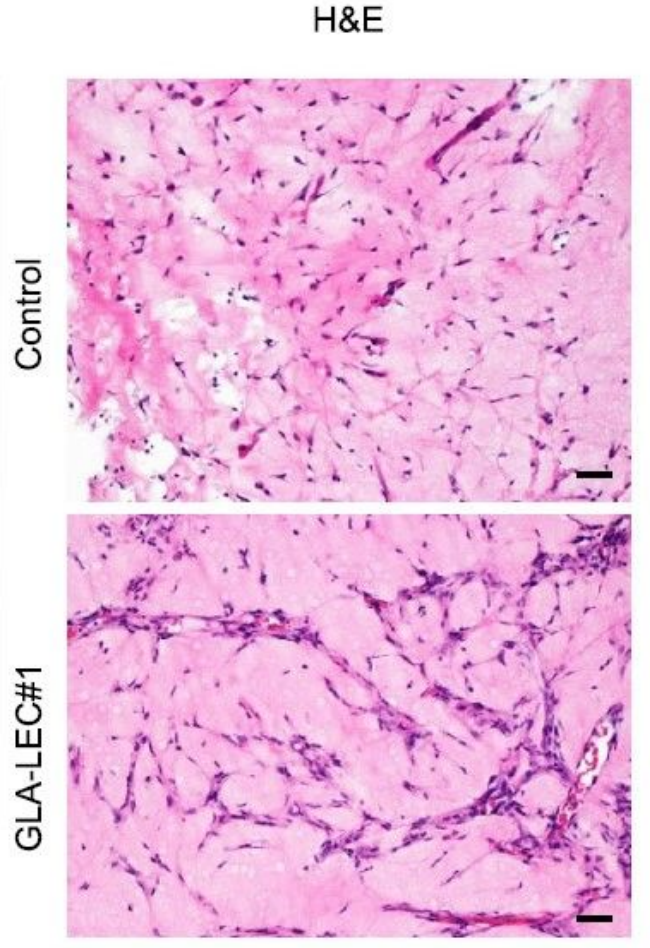

C

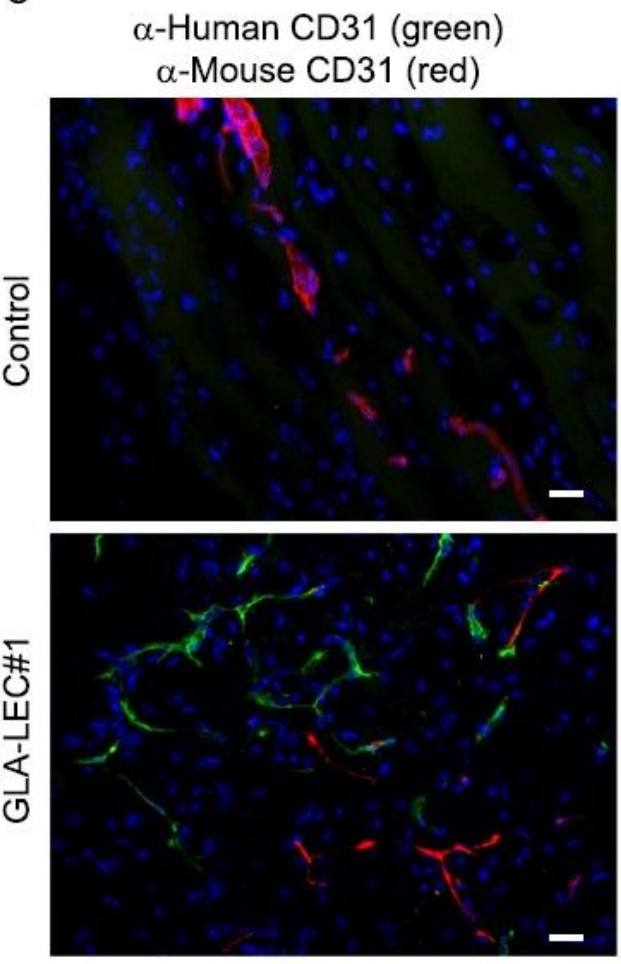

\section{Figure 4}

In vivo angiogenesis assay of GLA-LEC. (A) GLA-LEC\#1 $(2 \times 106)$ was injected subcutaneously into immunodeficient mice (NOD. Cg-Prkdcscid II2rgtm1Wjl/SzJ). After 10 days, Matrigel plug were removed from both control (Matrigel alone) and GLA-LEC\#1 group. (B) Hematoxylin and Eosin staining revealed that grafts with GLA-LECs contain robust vessel-like structure, but not in control sample. (C) To identify the patient derived GLALEC\# 1, immunoflurescence staining by anti-human CD31 (in green) and antimouse CD31 (in red) antibodies were conducted. In most of the vessel-like structure, endothelial cells are patient derived GLA-LEC\#1, but not host derived mouse endothelial cells. 


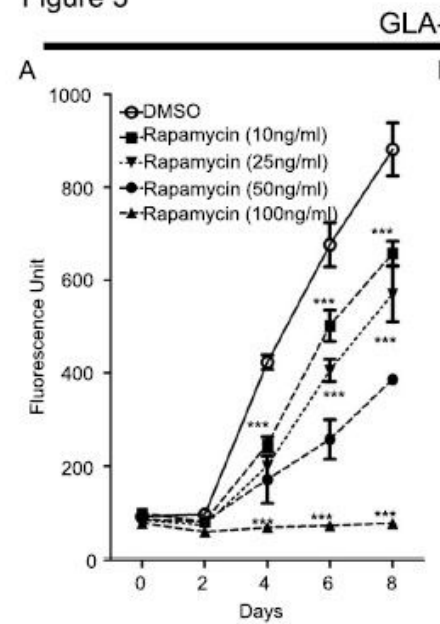

GLA-LEC\#1

C

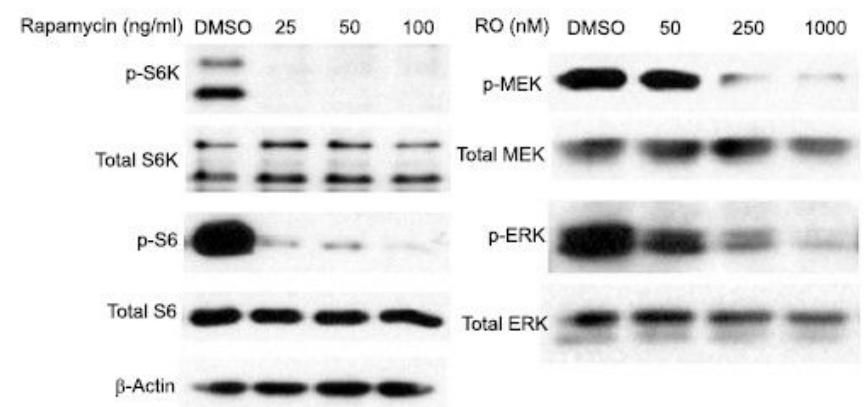

GLA-LEC\#2
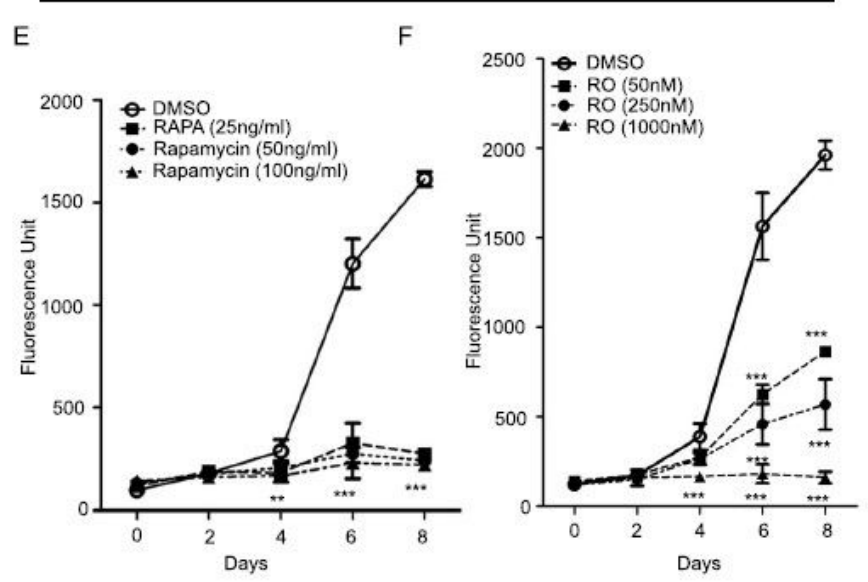

G

$\mathrm{H}$



\section{Figure 5}

GLA-LECs are sensitive to Rapamycin and MEK / ERK dual inhibitor treatment. (A, E) GLALEC\# 1 and GLA-LEC\#2 were treated by different dose of rapamycin $(10 \mathrm{ng} / \mathrm{ml}, 25 \mathrm{ng} / \mathrm{ml}, 50 \mathrm{ng} / \mathrm{ml}, 100 \mathrm{ng} / \mathrm{ml})$. CyQUANT assay was used to determine the cell proliferation. ${ }^{\star \star *} \mathrm{p}<0.001, \mathrm{~N}=3$. $(\mathrm{C}, \mathrm{G})$ Western blotting was 13 applied to study downstream signaling pathway involved in rapamycin treatment. (B, F) GLALEC\#1 and \#2 were treated by different dose of dual MEK / ERK inhibitor R05126766 (10nM, 50nM, $250 \mathrm{nM}, 1000 \mathrm{nM})$. CyQUANT assay was used to determine the cell proliferation. ${ }^{*} * \mathrm{p}<0.001, \mathrm{~N}=3$. Western blotting was applied to investigate the downstream signaling pathway involved in MEK / ERK inhibitor treatment. 
Figure 6

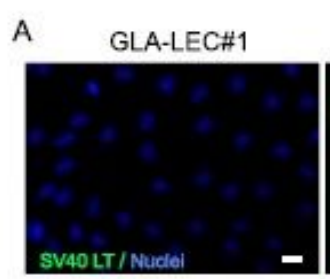

D



C



$\mathrm{F}$

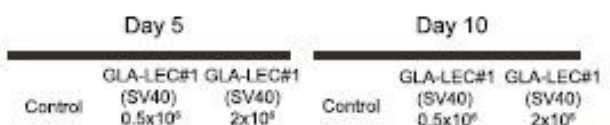

$\begin{array}{llll}\text { Control (SV40) (SV } 40) & \text { Control (SV40) (SV40) }\end{array}$

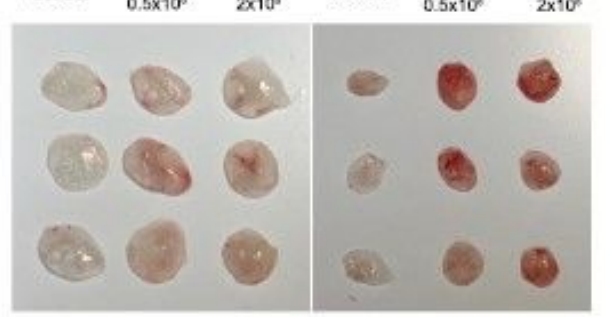

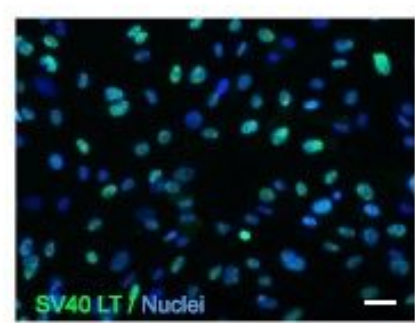

Passage 22
G
B

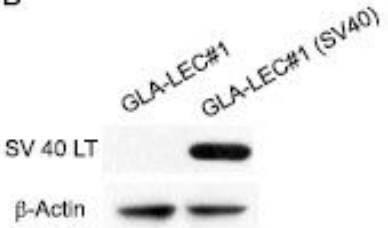

E

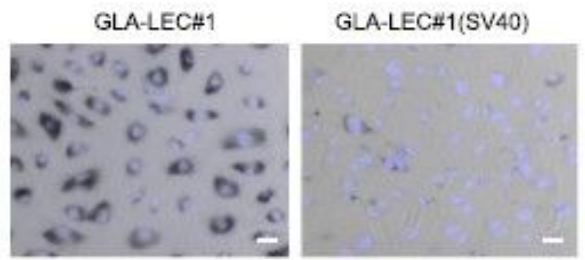

$\mathrm{X}$-gal / Nuclei

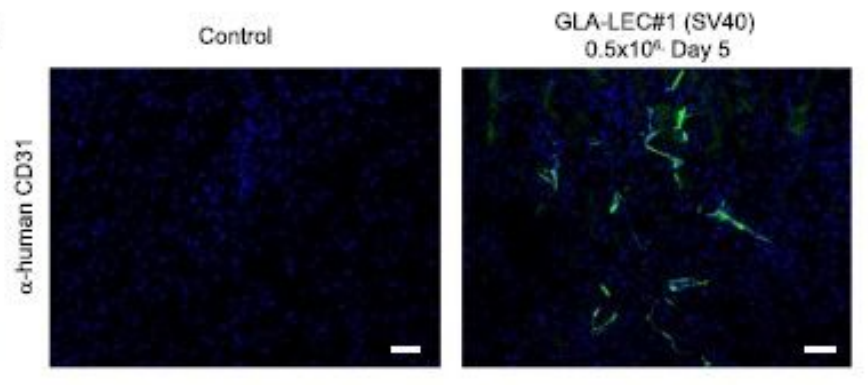

\section{Figure 6}

GLA-LEC\#1 immortalized with SV40 large T antigen. (A, B) Retrovirus was used to overexpress SV40 T antigen in GLA-LEC\#1. Both immunocytochemistry and western blotting were used to determine SV40 large T expression in the generated cell line. (C) Cumulated population doubling levels of the parental GLA-LEC\#1 compared with GLA-LEC\#1 (SV40). (D) Later passage (passage 22), SV40 LT antigen is still can be detected and stably expressed in the immortalized GLA-LEC (SV40) cell line. (E) In later passage (passage 22), GLA-LEC\#1 showed increased number of SA-X-gal-stained cells, whereas the GLA-LEC (SV 40) did not show any positive staining. $(F, G)$ In vivo GLA-LEC (SV40) Matrigel plug assay. With different cell density (0.5×106 and 2×106), different time point (Day5 and Day10), GLA-LEC (SV40) showed robust vessel-like structures detected by antihuman CD31 antibody immunoflurescence staining. Scale bar, 100冈m.

\section{Supplementary Files}


This is a list of supplementary files associated with this preprint. Click to download.

- TableS1.docx 